\title{
Contesting the Geneticization Thesis in Human Reproduction: Dealing with Spiral Shaped Processes and Technoscientific Imaginaries
}

\author{
Ido Alon ${ }^{a}$, Jose Guimon ${ }^{a}$, Rosa Urbanos-Garridob \\ Idoalon77@gmail.com; jose.guimon@uam.es; urbanos@ccee.ucm.es \\ ${ }^{a}$ Department of Development Economics, Research Group on Economics and Management of \\ Innovation, Autonomous University of Madrid, Spain \\ ${ }^{b}$ Department of Applied Economics, Public Economics and Political Economy, Complutense University of \\ Madrid, Spain
}

\begin{abstract}
:
This paper questions the potential shift of reproduction towards assisted reproductive technologies due to benefits provided by genetic manipulation of embryos. In order to examine the viability of such a shift and its implications from a regulatory perspective, we relied on two panels of experts from Israel and Spain, using the Delphi method and a series of in-depth interviews. We anticipate, at a first stage, a continuous-steady growth in the use of IVF, supplemented by preimplantation genetic diagnosis and the introduction of CRISPR/Cas. At a second stage, attracting a growing share of fertile people would require developments in genomics. While it is unclear whether these developments will fully materialize, they could be replaced by technoscientific imaginaries generating perceived benefits. We conclude that the regulation of reproductive genetics is becoming more critical and complex. The aim should be to ensure good practices and equity, while providing more information to the public. A broad and inclusive societal debate may overcome the difficulty of drawing a clear line between medical uses and non-medical uses of genetic selection and engineering and may contribute to finding the right balance between allowing autonomous decisions of patients and protecting the public interest.
\end{abstract}

Key Words: assisted reproductive technologies; In-Vitro Fertilization; Delphi; geneticization; Preimplantation Genetic Diagnosis; diffusion of innovation

\section{Introduction}

Louis Brown's birth in 1978 was the first successful In-Vitro Fertilization (IVF) for human reproduction, following 30 years of attempts. Since then, IVF has remarkably improved, providing solutions to an increasing number of pathologies of infertility. IVF is increasingly complemented through embryo selection by Preimplantation Genetic Diagnosis (PGD), available since 1990, and particularly by 
Preimplantation Genetic Screening (PGS) to identify chromosomal abnormalities. More recently, genetic engineering (GE) of human embryos by CRISPR/Cas has become practicable, although it remains at an experimental stage.

Long before these developments, in "Brave New World" (1932), Huxley had imagined a world where reproduction is mainly attained through Assisted Reproductive Technology (ART) due to the benefits associated with the selection and manipulation of embryos. Ever since, many other authors have developed this geneticization thesis and its implications for humankind (Ramsey, 1972; Silver, 1997; Greely, 2016). In this paper, we refer to the geneticization of reproduction as the process whereby the ability to select or design genetic traits of embryos in-vitro could turn IVF from a technical solution for infertility into the mainstream procedure for reproduction. Lippman defined the term geneticization as "an ongoing process by which differences between individuals are reduced to their DNA codes, with most disorders, behaviors and physiological variation defined, at least in part, as genetic in origin" (Lippman, 1991: p. 19).

Common speculation in the bioethics literature discusses the possibility that, at some point, the qualities of genetically selected or engineered persons will surpass those of naturally conceived ones, which would motivate a regular use of Reproductive Genetic Services (RGS) (Greely, 2016; Knoepfler, 2016). Such a scenario could be identified as the consequence of a "technology-push" diffusion trajectory of ART (Nemet, 2009), resulting from "a moment of breakthrough" (Brown \& Michael, 2003).However, in this paper, we depart from technological determinism or breakthrough perspectives and emphasize instead that technology and the social environment influence each other reciprocally in an extended process, shaped by the evolution of technological momentum and social values over time (Brown \& Michael, 2003; Nuffield Council on Bioethics, 2018). Indeed, the literature on technological change emphasizes that the process of innovation is not linear but interactive (Nemet, 2009; Di Stefano et al., 2012). Therefore, we complement the "technology-push" approach with a "demand-pull" perspective that underscores the importance of socio-technical imaginaries and pays particular attention to the role played by the regulatory framework (Nemet, 2009).

This paper reconsiders the viability of the geneticization thesis in human reproduction and contributes to the ethical discussion concerning ART, which holds important implications for regulation. We contribute to this agenda by critically analyzing the diffusion trajectories of ART and collecting keyinformants' views concerning the regulation of RGS through a series of in-depth interviews and Delphi surveys with panels of experts from two leading countries in the field, Israel and Spain. 
Delphi is a widely used qualitative method for forecasting, assessment and decision making regarding complex problems, built on a panel of experts who contribute with their knowledge and experience (Landeta et al., 2008). It is conducted with controlled feedback following a two-rounds survey, which allows experts to change their replies or add comments after learning the general views (Landeta \& Barrutia, 2011; Von der Gracht, 2012). Our Delphi panels included experts from various fields and aimed to simulate bioethics committees as those typically advising the regulatory processes.

Israel and Spain are among the most active ART industries in the world. In 2014, Spain was practicing the largest number of IVF cycles in Europe and a third of the continent's PGD procedures (ESHRE, 2018). Israel had the highest number of IVF cycles worldwide in relative terms (Health Ministry of Israel, 2018). Both are holding a very liberal RGS legislation, leading them to conduct venturesome PGD practices (Pavone \& Arias, 2012; Zuckerman et al., 2017; ESHRE 2018). Nevertheless, there are significant institutional and cultural differences between both countries, which influence their ethical conceptions towards ART and their regulation. Thus, these countries provide fertile ground for a comparative study concerning the geneticization of reproduction.

Building on inputs from Delphi surveys and interviews, and grounded on a previous technology forecast based on a panel of physicians (Alon et al., 2019), we discuss the potential trajectories for the diffusion of $\mathrm{ART}$, and present different scenarios which may assist in building up some anticipatory competence to enhance the regulatory process (Brown \& Michael, 2003; Borup et al., 2006; Harmon, 2016). This paper also contributes to broader studies on the diffusion of innovation, in particular to the strand of the literature that relies on qualitative methods to evaluate health and innovation policy by jointly considering supply, demand, regulation and the interactions among them (Nemet, 2009; Hammarberg et al., 2016).

\section{The geneticization of reproduction}

The geneticization of reproduction requires a significant increase in the scope of disorders treated by RGS. It involves a shift in the focus of genetic testing, from diagnosing monogenic disorders of early-onset and high level of penetrance (such as cystic fibrosis and sickle cell anemia), towards addressing less severe disorders of later onset and partial penetrance level (such as most metabolic, cardiovascular, cancerous and neurological diseases) (Klitzman, 2008; Batzer \& Ravitsky, 2009). Nowadays, PGD is already used for some polygenic diseases of late-onset and partial penetrance, such as neurodegenerative disorders and hereditary cancer (Altarescu et al., 2015; Dagan et al., 2017). Further diffusion of RGS would largely 
depend on their capacity to deliver enhanced children whose health is easier (and cheaper) to maintain (Greely, 2016; Knoepfler 2016).

Expectations, imaginaries, and fears occupy a pivotal role in the innovation process by shaping its potential (Brown \& Michael, 2003; Borup et al., 2006). Several authors have expressed high expectations regarding RGS in terms of human enhancement (Harris, 2007; Murphy, 2014). Conversely, intense preoccupations have been expressed concerning these technologies; particularly regarding undesirable collateral effects leading to epigenetic implications and future health consequences to those who descent from ART and their future generations (ESHRE, 2014; Fauser et al., 2014; Nuffield Council on Bioethics, 2018).

Moreover, it has been argued that the geneticization of reproduction would reduce human diversity and might lead to modern eugenics, i.e., the desire to enhance society with stronger, smarter and "better" people (Lippman, 1991; Garland-Thomson, 2015), resulting in social inequalities and discrimination (Buchanan et al., 2000; Fukuyama, 2002; Sandel, 2004). Furthermore, Silver (1997) has suggested that, in a distant future, a "geneticization arms race" could lead to polarization of society. Privileged societal groups would evolve so far through genetic enhancement that, at a certain point, the "gen-rich" groups would completely lose interest in mixing or sharing anything with the "regular" people.

From a more philosophical stance, the ambition to design children has been criticized as weakening instead of empowering, since by imposing our desires and beliefs on future generations, we would undermine their autonomy and deny their right for an open future (Ramsey, 1972; Jonas, 1984; Fukuyama, 2002; Habermas, 2003; Sandel, 2004). As anticipated by Lewis (1947, p. 37-39): “The final stage is come when Man by eugenics, by pre-natal conditioning (...) has obtained full control over himself. Human nature will be the last part of nature to surrender to Man. The battle will indeed be won. But who, precisely, will have won it?"

Nevertheless, at least three conditions must be met for further diffusion of RGS, which might materialize these extreme scenarios. First, technological developments ought to make IVF safer, more comfortable, and efficient. Second, RGS must introduce real or perceived medical or non-medical benefits in order to persuade the public to substitute natural reproduction. Third, regulations should be set in alignment with these developments and allow a broader portfolio of RGS.

Diffusion of innovation is a multi-cycle, two-way process of communication between different agents in society (Rogers, 1983), and often described as an interaction between supply and demand (Nemet, 
2009; Di Stefano et al., 2012). In the case of health technologies, Beck-Gernsheim (2000) describes a "spiral-shaped" process where technology is an effect and a cause simultaneously, stressing the influence of technology on values and needs. The relationship between regulation and innovation is neither static nor single-directional but instead reciprocal, since regulation affects innovation and, in turn, its outcomes create new conditions to be regulated (Paraskevopoulou, 2012). Innovation also alters the social values upon which regulation is based (Beck-Gernsheim, 2000). Furthermore, the "dual-process" theory enables us to distinguish between two cognitive routes shaping technology diffusion: "systematic processing", that relates to the conscious and observable improvement in outcomes (i.e. healthier babies), and "heuristic processing", which relates to the unconscious (i.e. the formation of social imaginaries created by desires and expectations) (Rommetveit, 2011; Jiahua et al., 2016; Tarkkala et al., 2018). The rest of this section develops a framework for analyzing the diffusion of ART from the supply, demand, and regulatory perspectives, as sketched in Figure 1.

Figure 1 - Interactions of supply, demand and regulations in ART.
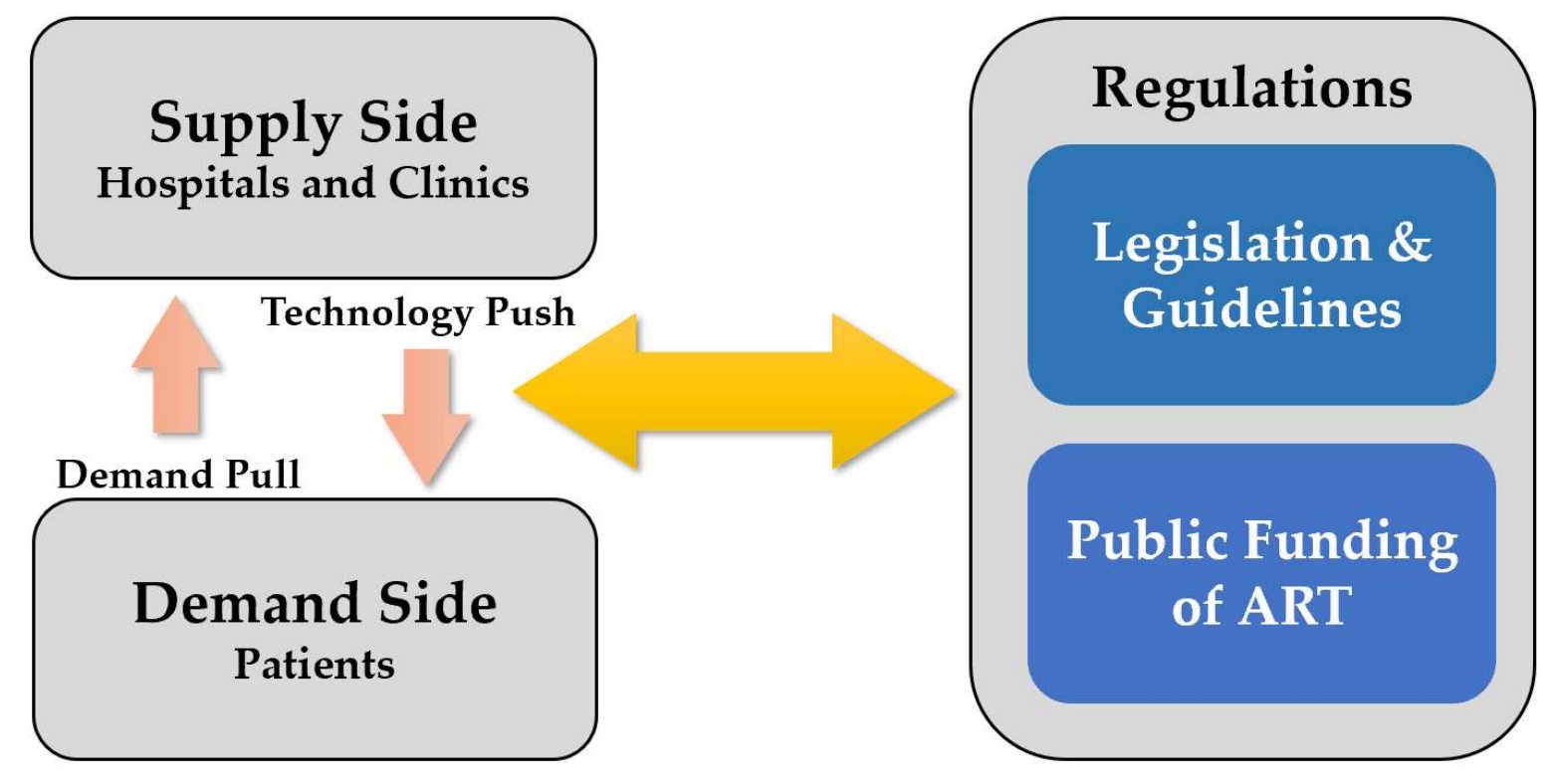

Source: own elaboration

\subsection{Technical factors}


In four decades, IVF has improved substantially, offering solutions to infertile couples, single women, same-sex couples, carriers of genetic disorders, and patients with a need or desire to preserve their gametes. For more far-reaching medicalization of human reproduction, IVF combined with RGS should enable to produce "healthier" children than natural reproduction. Nevertheless, such developments face some technical and social barriers.

IVF cycles begin with controlled ovarian hyperstimulation, a process which bears several health risks and inconveniences (La Marca \& Sunkara, 2014). It enables the ovaries to produce several eggs (with significant disparities between patient and cycles), which are then extracted (Cai et al., 2011). Subsequently, the retrieved eggs are fertilized by placing them in semen for fertilization to take place, or by injecting a single spermatozoon, directly into the cytoplasm of each egg thru intracytoplasmic sperm injection, which is used for the majority of IVF cycles in the U.S. and Europe (CDC, 2018; ESHRE, 2018). Only a part of these eggs become embryos available to be transferred to the uterus. According to different national registries, IVF is currently producing, on average, around $30 \%$ birth rates per cycle with nondonor eggs for women younger than 35 years, while success rates decrease in more advanced age groups (SEF, 2016; CDC, 2018). According to experts, we may expect significant growth in these rates, up to $50 \%$ within 20 years, due to improvements in methods of embryo selection, incubation, laboratory conditions and quality control (Alon et al., 2019).

The addition of PGD requires extracting DNA from a biopsy taken from each embryo before implantation, which is then diagnosed for pre-identified mutations. Currently, PGD requires an extensive pre-study of family members to identify a single mutation associated with a severe disorder (Altarescu et al., 2015), which constitutes a labor-intensive, customized procedure (Wang, 2014; Swanson et al., 2007). In recent years, next-generation sequencing has been introduced, potentially allowing to infer the full genome sequence of every embryo in a more efficient manner (Nuffield Council on Bioethics, 2018). Nevertheless, PGD exposes the embryos to some additional risks, since a biopsy is an invasive procedure involving extracting cells from the embryo, and it also demands to leave the embryo longer in the incubator until a biopsy may be extracted.

Biopsies can also be used for PGS, a global quantitative analysis of the entire genome which serves to detect and transfer only euploid embryos (i.e., without chromosomal structure anomalies). This procedure assumes that an euploid embryo has better chances to develop into a fetus and a healthy baby (Lu et al., 2016; Casper et al., 2017). PGD cycles represent a minor share of all IVF cycles, while PGS is more 
commonly used and mainly offered to patients of advanced age whose eggs tend to have higher levels of aneuploidy.

Next-generation sequencing also enables whole-exome sequencing. The exome makes up only $1.5 \%$ of the whole genome, but it contains all protein-coding genes. It has been estimated that more than 10,000 monogenic disorders affect around one percent of humans at birth, and about two percent of couples carry a single gene variation that could result in a child with a severe genetic disorder (Aslamkhan, 2015; Babar, 2017; Nuffield Council on Bioethics, 2018). However, the detection pace of new monogenic disorders is declining while, in contrast, detection pace of polygenic disorders and multifactorial traits, which affect a much larger share of the population, is increasing (Nuffield Council on Bioethics, 2018; Second International Summit on Human Genome Editing, 2018a). It means that, with further developments in genomics, we may expect a higher utility of PGD, although according to experts, it will primarily be related to elderly diseases which are mainly multifactorial (Alon et al., 2019).

Performing an "expanded PGD" for various complex polygenic or multifactorial disorders would require a dramatic increase in the number of fertilized eggs, to enable detecting a "perfect" embryo. It will demand patients to overgone many more cycles and expose them to increased medical risks. Some experts suggest that such an abundant supply of eggs could be attained by stem-cells derived gametes (Shulman \& Bostrom, 2014; Greely, 2016), but this is yet an unclear technological enhancement. Overall, expert geneticists and gynecologists have shown low expectations for significant technological advances in eggs retrieval in the next two decades (Alon et al., 2019).

Alternatively, advances in GE, and mainly the introduction of CRISPR/Cas, may enable a much broader manipulation of human genetic traits. Developed in 2012, CRISPR/Cas is a simple, low-cost tool for gene editing which enables to cut strands of DNA. With CRISPR/Cas only a few embryos would be required, and for each embryo, various DNA fragments could be edited. However, CRISPR/Cas is still an emerging technology subject to considerable uncertainty. Germline modification carries significant risks of unintended side effects, which remain unclear. Moreover, once a gene edit is introduced, there is currently no method to remove it (Evitt et al., 2015; Nuffield Council on Bioethics, 2018; van Dijke et al., 2018).

Lastly, despite the expected technological improvements in IVF, implanting an embryo in the uterus is not a guarantee for success. Therefore, following the use of PGD or CRISPR/Cas, transferring the selected or designed embryo into the uterus will provide no promises of live birth. 


\subsection{Demand for ART}

IVF births already approximate or exceed $5 \%$ of the total in several countries (SEF, 2016; ESHRE, 2018; Health Ministry of Israel, 2018). It has been fueled by the prevalence of infertility, which currently affects $10-15 \%$ of the human population (Agarwal et al., 2015; ASRM, 2015) and has been rising due to environmental hazards and unhealthy lifestyles (Inhorn \& Patrizio, 2015; Sobotka, 2016). Notably, the rise in the average age of parenthood has been framed as a risk factor (Pavone, 2010), increasing the popularity of embryo selection by PGD/PGS, which already accounted for $22 \%$ of IVF cycles in the U.S. in 2016. According to different estimations, infertility affects $10-15 \%$ of the human population (Agarwal et al., 2015; ASRM, 2015). Moreover, the use of ART for social reasons has gained public consent in many countries, expanding the demand from women beyond the age of fertility (by egg donation or by eggs cryopreservation), single women and same-sex couples.

Nonetheless, most people are fertile, tend to reproduce early enough, carry no significant genetic disorders, and settle for prenatal genetic testing to avoid the birth of children with severe disorders. Would demand then face a glass ceiling? For further diffusion, ART must address new channels of demand. On top of the facilitation of treatment, improvements in RGS must bring along promises of significant benefits to the public. Proven health benefits concerning less severe polygenetic disorders and diseases (which appear at a lesser level of penetrance, with later onset, and affect a much larger share of the human population), would constitute the conscious aspect of this dual process. However, such benefits could take a long time to be demonstrated, if at all. Therefore, at the unconscious facet of the dual-process stands the creation of socio-technical imaginaries induced by market forces, which may breed desires, expectations and new perceptions of health benefits that would in turn develop into needs and requirements, and later into parental and social responsibilities (Rommetveit, 2011; Tarkkala et al., 2018).

Recent demand forecasts (Alon et al., 2019) suggest that within 20 years, the percentage of IVF births could reach more than $14 \%$ in Israel and Spain, mainly due to age-related infertility. Moreover, approximately $40 \%$ of IVF cycles could involve PGD, meaning that, while it might not be the primary factor inducing the demand for IVF, PGD would become a very common add-on to treatments.

\subsection{Regulation}

Embryo selection by PGD began as a very controversial technology, raising ethical concerns regarding the deliberate waste of human embryos, the "playing God" argument, and the risk of a "slippery slope" (Zuckerman et al., 2017). Nevertheless, PGD has been progressively gaining consent, as it allows parents 
carrying genetic disorders to bring a healthy child into the world, while many seek such solution following the birth of a child affected with a severe genetic disorder. After all, PGD saves a great deal of emotional and financial resources associated with caring for a child with a "life not worth living", i.e., with very short life expectancy, intense medical care demands, and very low quality of life (Buchanan et al., 2000).

In most countries, PGD is highly regulated and practiced in accordance with the characteristics of genetic conditions under diagnosis (the severity of the disorder, monogenic or polygenic, early or late onset, high or low level of penetrance, curable or non-curable). Originally, PGD was used mainly for severe, non-curable monogenic disorders of full penetrance and early onset. However, RGS evolves following a spiral-shaped process, whereby values and needs boost the development of technology and its cultural acceptance, while technological change redefines values and needs (Beck-Gernsheim 2000).

At first, the use of PGD to avoid the birth of a child with an extremely short life expectancy was easily justified. Later, its success increased the level of public consent also in less extreme scenarios, and the availability of the technology has produced higher acceptance to use it for a growing set of disorders. In a previous study, although most experts supported many sorts of PGD, they drew a clear red line only between medical and non-medical uses of the procedure, showing a strong opposition against applications of RGS to identify and select physical or cognitive traits (Alon et al., 2019). Nevertheless, PGS is also gaining medical justification and social consent, thus becoming more frequently practiced in order to increase IVF success rates (Batzer \& Ravitsky, 2009; Pavone \& Arias, 2012).

Moreover, at the next step of RGS, CRISPR/Cas could provide a more efficient and effective solution. Trials on human embryos have been a growing success, but there is a "tacitly agreed" temporary moratorium on implanting genetically edited embryos into the uterus (Evitt et al., 2015). This consensus, however, was recently broken unexpectedly by one researcher in China, with uncertain consequences (Krimsky, 2019). Following this event, discussions at the $2^{\text {nd }}$ International Summit on Human Genome Editing, concluded that three conditions are required to approve the use of CRISPR/Cas on human embryos: (1) scientific rationale (medical justification), (2) safety and (3) social acceptance (Second International Summit on Human Genome Editing, 2018b), which currently are not fulfilled.

\section{Methods}

We conducted a Delphi consultation with a panel of experts from different fields related to ART, simulating two typical advisory committees (from Israel and Spain). We began by conducting semi- 
structured personal interviews with 29 experts to assist in building a Delphi questionnaire. These interviews also served to gather broader qualitative insights and to detect more participants based on recommendations by the interviewees, propitiating a "snowball" effect (Ribeiro \& Quintanilla, 2015).

Participants were selected based on their skills, experience, and unique contribution to public discourse (Lock, 1990). For this purpose, we consulted members' lists of the Spanish bioethics committee, and the latest (2012) government appointed Israeli "Mor-Yosef" committee (since in Israel advisory committees are occasionally appointed). The panel included 18 Israelis and 18 Spaniards, 21 women and 15 men. We selected experts of a multidisciplinary character as common in bioethics committees (see Bagheri et al., 2016; Gomes de Oliveira et al., 2017). All experts had interest in the ethical debate and their careers were dedicated to ART from the fields of medicine (12), law and bioethics (7), public health (5), psychology (3), biology (2), philosophy (2), economics (2), epidemiology (1), theology (1) and journalism (1).

The questionnaire dealt with general attitudes towards regulations, and with specific practices of RGS. It was based on 10-point scale questions and was complemented with open spaces for comments. The Delphi survey was limited to two rounds regardless of the degree of consensus achieved (measured by the coefficient of variation/standard deviation), a methodologically sound practice (Von der Gracht, 2012; Dayé, 2018). Between the rounds, we highlighted for each expert those answers which significantly differed from the central tendencies and asked regarding inconsistencies or substantial deviation from the group, using controlled feedback (Landeta et al., 2008; Skirton et al., 2013). The participants were offered the option to change their replies or add explanatory comments regarding their deviant positions. This method provides either consensus or qualitative insights to explain dissensus, which may assist in constructing alternative scenarios (Landeta \& Barrutia, 2011; Melander, 2018).

\section{Results}

\subsection{General attitudes towards regulations}

Focusing on the spiral-shaped process of RGS, we introduced four different statements and asked the panels to mark their level of support (from 1 -completely opposing- to 10 -completely supporting-). The answers are shown in Table 1.

Table 1. General attitudes towards the regulation of PGD 


\begin{tabular}{|c|c|c|c|c|}
\hline \multirow[t]{2}{*}{ From 1 (completely opposing) to 10 (completely supporting) } & \multicolumn{2}{|c|}{ Israel (n=18) } & \multicolumn{2}{|c|}{ Spain $(n=18)$} \\
\hline & Mean & SD & Mean & SD \\
\hline $\begin{array}{l}\text { a. The public sector should aim at reducing regulation regarding PGD to a } \\
\text { minimum in order to allow the patients maximum free choice. }\end{array}$ & 3.4 & 2.83 & 3.7 & 2.79 \\
\hline $\begin{array}{l}\text { b. When performing PGD for medical reasons, sex selection as an add-on } \\
\text { service should be allowed. In-other-words, the physician may reveal the sex of } \\
\text { the (clinically) selected embryos enabling the patients to choose. }\end{array}$ & 2.8 & 2.76 & 2.2 & 1.63 \\
\hline $\begin{array}{l}\text { c. When performing IVF due to infertility, regulation should be more tolerant } \\
\text { towards PGD. It should be allowed as an add-on service to IVF for some range of } \\
\text { disorders. }\end{array}$ & 7.9 & 2.51 & 6.3 & 2.66 \\
\hline $\begin{array}{l}\text { d. It is viable in terms of regulation to separate between the use of PGS and the } \\
\text { use of PGD for detecting disorders. In other words, in case PGS will eventually } \\
\text { become a very common add-on for IVF cycles to increase the prospects of the } \\
\text { treatment, regulation may still prevent the clinics from regularly using these } \\
\text { biopsies for PGD. }\end{array}$ & 4.8 & 2.25 & 5.6 & 2.37 \\
\hline
\end{tabular}

The experts did not support the liberalization of RGS. Although most supported the promotion of PGD to minimize future genetic diseases, they also believed that regulation should reduce unexpected risks. As stated by one of the interviewees:

"In the long run, we may find that by trying to prevent cancer by $P G D$, we increased the incidence of other cancers or malformations (exposing the fertilized egg to laboratory conditions). Not enough years have passed to make us confident in the safety of these techniques."

Some experts marked the importance of reducing asymmetric information, emphasizing that the technology is very sophisticated, and the public is not familiar with all its implications:

"Regulation should ensure safe and evidence-based services, make sure the offer of PGD is accompanied by appropriate counseling, and that the important decision to perform IVF for the sake of $P G D$ is fully informed and free of pressure".

"We should prevent society from falling into the false belief that RGS assures 100\% healthy offspring (...) we should not fall into genetic determinism when many other factors can influence people's health". 
Although most experts supported regulating the field, some advocated the consideration of personal autonomy as much as possible, urging to "avoid heavy-handed regulation of PGD and leave the reproductive decision making to women and couples, based on a principle of reproductive autonomy". However, it was also stated that "patients" choice and their consent for therapies should take place within a normative framework and a public health system. Therefore, the freedom of choice cannot be total".

The experts opposed revealing gender upon performing PGD. Comments emphasized the fear that allowing it would turn every PGD into a sex-selection. In contrast, PGD as an add-on to IVF was approved by the panels (more strongly by the Israeli), which underlined again the priority given to preventing medical disorders. Several experts raised the slippery-slope argument in their open comments:

"The definition of 'a range of disorders' is very vague... where will it lead us? Where are the boundaries?"; "Why for a variety of genetic diseases rather than genetic anticipation or nextgeneration sequencing?"

"The process of selecting 'perfect' embryos occurs gradually, and we become accustomed to the idea so that later it will seem natural to prevent the birth of infants with treatable diseases or even traits that we have no reason to prevent".

Finally, the experts were doubtful regarding the ability to stretch a line between PGD and PGS in case the latter becomes an official add-on to IVF. In the words of one of the experts: "There is a regulatory weakness in this regard because the line is very loose. Where settings are not sharp, regulation will lose...".

\subsection{Regulation of specific practices of RGS}

Table 2 presents experts' attitudes towards specific applications of RGS. We distinguish three different levels of consent: strong support, mild support, and disapproval, each marked with a different color in the table.

Table 2. Attitudes towards the regulation of specific RGS practices

\begin{tabular}{|c|c|c|c|c|c|}
\hline \multirow[t]{2}{*}{ From 1 (completely opposing) to 10 (completely supporting). } & & \multicolumn{2}{|c|}{ Israel $(n=18)$} & \multicolumn{2}{|c|}{ Spain $(n=18)$} \\
\hline & & Mean & SD & Mean & SD \\
\hline \multirow{2}{*}{$\begin{array}{l}\text { a. PGD for severe monogenic disorders of early-onset and high- } \\
\text { level of penetrance with no simple cure. }\end{array}$} & Allow & 9.6 & 0.62 & 9.3 & 2.20 \\
\hline & Fund & 9.5 & 0.64 & 8.9 & 2.30 \\
\hline \multirow{2}{*}{$\begin{array}{l}\text { b. PGD for severe monogenic disorders of medium-late onset and } \\
\text { a high level of penetrance with no simple cure. }\end{array}$} & Allow & 9.4 & 0.81 & 8.9 & 2.19 \\
\hline & Fund & 9.2 & 0.94 & 8.5 & 2.35 \\
\hline
\end{tabular}




\begin{tabular}{|c|c|c|c|c|c|}
\hline \multirow{2}{*}{$\begin{array}{l}\text { c. PGD for severe monogenic disorders of medium-late onset and a } \\
\text { medium level of penetrance with no simple cure. }\end{array}$} & Allow & 7.5 & 2.22 & 8.6 & 2.43 \\
\hline & Fund & 6.9 & 2.29 & 8.1 & 2.60 \\
\hline \multirow{2}{*}{$\begin{array}{l}\text { d. GE (CRISPR/Cas) for severe monogenic disorders of early-onset } \\
\text { and high-level of penetrance with no simple cure in case PGD did } \\
\text { not provide a solution. }\end{array}$} & $\overline{\text { Allow }}$ & 7.5 & $2 . \overline{29}$ & 6.4 & $3 . \overline{39}$ \\
\hline & Fund & 6.5 & 2.79 & 4.8 & 3.51 \\
\hline \multirow{2}{*}{$\begin{array}{l}\text { e. PGS for detection of chromosomal abnormalities, in order to } \\
\text { increase the prospects of an IVF treatment. }\end{array}$} & Allow & 6.9 & 2.79 & 6.2 & 3.29 \\
\hline & Fund & 5.7 & 2.97 & 4.3 & 3.24 \\
\hline \multirow{2}{*}{$\begin{array}{l}\text { f. PGD for multifactorial diseases, (cancerous/ metabolic/ } \\
\text { cardiovascular/ neurological) with medium-late onset and medium } \\
\text { level of penetrance. }\end{array}$} & Allow & 6.4 & 2.60 & 6.0 & 3.28 \\
\hline & Fund & 5.2 & 2.34 & 4.3 & 2.66 \\
\hline \multirow[t]{2}{*}{ g. PGD with whole-exome screening. } & Allow & 3.8 & 2.29 & 4.3 & 3.15 \\
\hline & Fund & 2.6 & 2.22 & 3.1 & 2.82 \\
\hline \multirow[t]{2}{*}{ h. PGD for social sex selection. } & Allow & 2.1 & $1 . \overline{88}$ & 3.7 & $3 . \overline{37}$ \\
\hline & Fund & 1.8 & 1.51 & 1.4 & 1.42 \\
\hline \multirow[t]{2}{*}{ i. PGD for cognitive characteristics selection. } & Allow & 1.3 & 1.19 & 1.5 & 1.29 \\
\hline & Fund & 1.4 & 1.22 & 1.3 & 0.96 \\
\hline \multirow[t]{2}{*}{ j. PGD for physical traits selection. } & Allow & 1.3 & 1.19 & 1.3 & 0.97 \\
\hline & Fund & 1.4 & 1.22 & 1.3 & 0.96 \\
\hline
\end{tabular}

\section{Strong support (green zone)}

The experts strongly supported, with a high level of consensus, the use of PGD for monogenic disorders of a high level of penetrance either with early or late onset. However, there was a lower consensus regarding the use of PGD for disorders of medium level of penetrance (including many cancerous diseases), with the Spanish panel expressing stronger support than the Israeli. Despite the lack of consensus, both panels tended to support allowing and funding it. As stressed by one interviewee: "We are not talking here about curable diseases. Some genetic disorders of 'medium level of penetrance' are in practice devastating cancers which may affect various family members".

Experts mostly supported the coverage of PGD by the public system in order to avoid health inequalities and guarantee fair and inclusive access to the services.

\section{Mild support (grey zone)}


The panel displayed less consensus regarding four contested categories for which health benefits are not yet clear. First, concerning CRISPR/Cas for germline editing, we presented the panel with a specific case referring to a severe disorder of early-onset and high level of penetrance where PGD cannot be delivered due to a shortage of eggs/embryos. The panel expressed reasonable support in this case, and the main preoccupations were about safety, beneficence principle, efficiency, and low cost. The slipperyslope argument was not raised.

Second, with respect to PGS for chromosomal abnormalities, some experts emphasized the undemonstrated usefulness or cost-effectiveness of the technique. For example, one interviewee argued that "PGS is contraindicated in the following cases: advanced maternal age, early ovarian failure, low response, poor embryo quality, severe male factor, and more". Other experts expressed preoccupation regarding the increased use of PGS, claiming that it is occasionally offered as an add-on to IVF cycles, increasing the financial burden on patients only to produce gains to private clinics.

Third, regarding PGD for multifactorial diseases, the experts distinguished between diseases for which there is a genetic cause with a reliable diagnosis, where PGD should be allowed and financed and other multifactorial diseases which cannot yet be diagnosed by PGD and therefore should be strictly regulated. One expert claimed that "there are no immediate perspectives that PGD contributes anything significant regarding disorders involving more than a single gene or when a larger number of factors cause a disease".

Fourth, the experts did not express high support for using PGD for whole-exome screening. Qualitative comments from interviews help understand these results:

"I reject the use of techniques that are not directly preventing or treating diseases, for which safety is not guaranteed or whose impact and consequences on the human species are yet unknown".

"The problem is that there will always be some suspicious mutations (although the emphasis will be given only to areas where there is a family history or where a gene for carriers has been identified), but if each finding were revealed to the patient, there would be no embryo remained to implant".

Differences between "allowing" and "funding" the procedures were noticed, as illustrated with the following quotes from our interviewees:

"The public system, always with limited resources, should prioritize the financing of other health needs, rather than PGD for multifactorial diseases, which could lead to an uncontrollable demand". 
"Allowing these procedures would enable autonomy for couples in decisions concerning their children's health. Another issue is financing these procedures with public funds when we have many other urgent priorities regarding health issues that are not subjected to probability."

\section{Disapproval (red zone)}

Finally, PGD for non-medical needs was discarded by the experts. As argued by one interviewee:

"The ethical boundary regarding the application of PGD should be between avoiding hazards and satisfying preference or choice of characters, simply according to the parent's preference and not for the future benefit of an individual. I can accept the application of scientific knowledge to avoid suffering but not to satisfy whims".

Nevertheless, a few comments from the panel were tolerant towards sex selection: "it should be considered in some particular cases (couples with few children of the same gender who are psychologically affected by the lack of offspring of the other gender)...it is not necessary to criminalize them"; and specifically, regarding Israel: "Sex selection in this area of the world is sometimes much more than merely 'social' and understandably allowed if having the 'wrong' sex baby might jeopardize either the mother or the baby".

\section{Discussion}

Despite the different cultural and institutional profiles of Israel and Spain, both panels converge in that any use of ART which is safe enough and provides significant health benefits should be approved. The experts awarded health and individual autonomy the highest importance, although they remained attentive to some potential social risks.

Based on the main results of the Delphi panels described in Section 4, supplemented by our previous research focused on technological forecasting (Alon et al., 2019), we proceed to evaluate the extent of geneticization of human reproduction by presenting two foreseeable stages with two plausible scenarios at the second stage.

Stage 1. RGS as an add-on to IVF: This stage of geneticization is, to a great extent, already realized today in Israel and Spain. In the following decades improvements in IVF will resolve more pathologies of infertility. Demand for IVF will increase due to the postponement of parenthood, the rise of age-related 
infertility, and fertility preservation. Environmental factors, unhealthy lifestyles, the use of PGD, and elective uses of IVF will also spur demand (Alon et al., 2019).

Meanwhile, PGS is already becoming a more standard add-on. In Spain, where the number of PGD/PGS cycles has tripled between 2010 and 2016, more than $80 \%$ of these cycles were referred to PGS (SEF, 2010; SEF, 2016). Furthermore, biopsies extracted for PGS will increasingly be used also for PGD, which will enable detecting a wider variety of disorders; multifactorial, of later-onset, lower level of penetrance and even less severe expressions, providing higher accuracy and success rates (Lu et al., 2016).

Later at this stage of diffusion, clinical GE of human embryos will be introduced, beginning with some cases where PGD fails to deliver. ${ }^{1}$ Both panels were positive regarding the application of CRISPR/Cas in embryos for therapeutic means, following a further societal debate and a growing international consensus (see also Second International Summit on Human Genome Editing, 2018b; van Dijke et al., 2018). Unless inappropriate use with disastrous results generates public mistrust in this technology, CRISPR/Cas will eventually be perceived as more practical and efficient than PGD. Nonetheless, it is likely to be a long and gradual process, and even after the first applications of CRISPR/Cas, preoccupations with safety and adverse effects will take time to dissolve.

Potentially, with CRISPR/Cas breaking through, a much wider variety of possibilities will be opened, from replacing whole genes to fixing aneuploid embryos, and even aimed at curing complex multifactorial diseases. CRISPR/Cas might be revolutionary or age defining, but it is yet early to measure the relative impact of genetic factors on the many characteristics that people may wish to influence. It is therefore hard to foresee the potential uses of genome editing, and it might as well be limited for an extended period to single-gene disorders and a few more conditions of limited complexity (Nuffield Council on Bioethics, 2018.

At the end of stage $1,15-20 \%$ of births in these countries will be due to IVF, and about $40 \%$ of cycles will include RGS (Alon et al., 2019). However, the use of RGS will still significantly benefit a limited share of the population (those with a medical history of hereditary diseases).

\footnotetext{
${ }^{1}$ The possible uses would be: Y-chromosome defects; inversions and deletions of chromosome segments; dominant genetic conditions such as Huntington's disease, some forms of Alzheimer's disease or breast cancer, where one of the prospective parents is homozygous; recessive genetic conditions where both parents are homozygous (Nuffield Council on Bioethics, 2018).
} 
Stage 2 - RGS for the mass: After maximizing the potential of ART as a solution to infertility, the only channel for further market growth will be RGS. However, the use of ART may carry risks for congenital damage, cancerous and cardiovascular diseases, developmental deficiencies, and cognitive disorders (Fauser et al., 2014; ESHRE, 2014). Similar worries regarding CRISPR/Cas have been raised (Evitt et al., 2015). Further evidence, proofing or refuting these concerns, will have significant weight on the second stage of diffusion since referring fertile couples with "good genes" to RGS will most likely be discarded in case high risks are perceived. At this point, we envision two divergent scenarios:

a) A market for desires: New findings in genomics may enable expanded PGD and CRISPR/Cas for multifactorial diseases and traits. However, most children are healthy, and most people do not suffer severe health conditions during their early and mature lives. Driving the public to approach RGS could be done mostly by promising to deliver children who will be more resistant to elderly diseases, or by enabling the selection of physical and cognitive traits, which the panels strictly rejected. Therefore, for most people, the greatest advantage of extended PGD would be related to late-onset multifactorial diseases (Alon et al., 2019).

Nonetheless, from a medical perspective, it may take a lifetime to prove benefits from an expanded PGD which aims at such diseases. It would also require follow-up throughout the adult lives of ART babies, a task with which scientists are already struggling today. In the meantime, evidence could be replaced by scientific speculations, beliefs, and expectations, with socio-technical imaginaries generating perceived benefits of geneticization.

Once physicians and patients are encouraged by supportive scientific evidence and regulators, become aligned, the second stage will kick-off. Competition between "embryo designers" will thrive, and the promise of a "perfect baby" will nourish the deepest desires of parents. RGS will ultimately turn into parental responsibility and end up as a social norm.

b) A limited market: Alternatively, despite the many technological developments, the remarkable improvements in IVF outcome and the benefits of RGS, more than $80 \%$ of the people would not require ART. They are fertile, opt to give birth early enough, and find no specific motivations to seek a genetically selected or engineered baby. After all, we can expect improvements not only in reproductive medicine but also in other fields. Why should people approach ART despite the physical and financial burden, only to design a baby resistant to complex diseases which could be cured or even prevented in a more efficient way? 
As explained previously, the share of the human population affected by monogenetic or relatively simple polygenetic disorders is quite limited (Aslamkhan, 2015; Babar, 2017). If RGS aims at moving towards multifactorial diseases and traits, it will require a better understanding of the complex and flexible interaction between genomics, environmental factors, and epigenetic alterations. Multifactorial diseases also involve more cases of gene pleiotropy (i.e., when one gene controls the phenotype or expression of several unrelated traits) (Nuffield Council on Bioethics, 2018). These complications could place severe limitations to RGS, as we may finally comprehend and accept the falseness of genetic determinism. (Buchanan et al., 2000; Ravitsky, 2002; Pavone, 2010).

Our work is not exempt from limitations. A Delphi panel selection always has a certain level of subjectivity: Our sample is not representative, nor exhaustive, and different experts may express distinct attitudes. Moreover, we selected countries which stand at an advanced stage of diffusion, have very proART attitudes, and tend to nourish a comprehensive public healthcare system. Different results might be obtained from countries where there is a strong attachment to individualism and a free-market economy. Nevertheless, the analysis introduced in this paper is compatible with recent literature (Pavone, 2010; Lu et al., 2016; Casper et al., 2017; Nuffield Council on Bioethics, 2018).

\section{Conclusion}

The medicalization of reproduction is a long and observable process, beginning with the growing practice of IVF, currently mainly driven by infertility. At this stage, RGS may be further introduced as an add-on, based on a dual process where, on the one hand, real outcomes are being produced and, on the other, imagined or uncertain outcomes are being perceived. After reaching a critical mass and generating more confidence, a second stage may arrive where genetic selection and engineering could become the market's main growth engine.

The geneticization of reproduction will therefore not result from a "moment of breakthrough" (Brown \& Michael, 2003) in a particular research project, but rather from a spiral shaped-process, influenced by gradual technology advances, socio-technical expectations and imaginaries, and shifts in public values. In this process, regulation interacts with supply and demand, holding a key role in directing ART trajectories to the benefits of society.

Our empirical study in Israel and Spain unveils that the moral justification of using ART for infertility and prevention of genetic disorders stands firm. The consulted experts drew a line between medical uses 
and non-medical uses of RGS, but there is a significant grey-zone, which is likely to expand and complicate the regulator's task as new technological developments materialize. Particularly as the Spanish law leaves great room for future extension of the definition of genetic diseases (Pavone, 2010), and Israel holds significant flexibility in this regard, having crucial decisions decentralized and handled by local committees in hospitals and clinics (Shalev \& Hashiloni-Dolev, 2011). At the first stage, the primary roles of regulation are to assure good practices and equity, correct information failures, and verify the collection of data to enable a more accurate follow-up and research. However, as evident from Israel, public policy may also promote the medicalization of reproduction by generously funding IVF cycles to support fertility rates. Concerning the possibility to step into the second stage of diffusion, and given the weight assigned by experts to medical justification, if the benefits produced by RGS will be supported by evidence, the way towards further medicalization of reproduction will be paved. Although such a scenario may still be far, the ART industry might gradually seek to expand by promising those unproven benefits, as in the case of PGS today (Orvieto \& Gleicher, 2016; Casper et al., 2017).

This leads us to conclude that despite the large economic potential of the ART industry, regulators should aspire to slow down the medicalization process. It could be achieved by promoting the prevention of infertility and by better informing the public regarding infertility and ART outcomes to avoid excessive expectations (García et al., 2017; Fauser et al., 2019). As ART is increasingly becoming a common way of reproduction, governments must enhance public awareness by disseminating clear information on its opportunities and risks.

Finally, as ART further develops, the importance of broad and inclusive international debate is growing. We may greatly doubt whether regulations in Israel, Spain, or any other country are ready to welcome the stage of advanced RGS. In order to be better prepared, it is essential to enhance regulatory collaboration between countries, since state borders and national regulations will play a decreasing role at the second stage (see also Martin, 2014). We should avoid "sleepwalking" into this process by allowing uncontrolled technological momentum (Nuffield Council on Bioethics, 2018). However, we should also prevent basing our ideology on outdated and misleading contexts. In other words, preventing RGS for non-medical reasons is not a total guarantee for stopping geneticization and, similarly, allowing patients to take autonomous decisions will not necessarily lead to social catastrophe. An open debate should address questions of freedom of choice and personal autonomy while being regularly updated according to the most realistic and accurate scientific context, to avoid falling into inflated hopes or dystopian theories. 


\section{References}

Agarwal, A., Mulgund, A., Hamada, A., \& Chyatte, M. R. (2015). A Unique View on Male Infertility Around the Globe. Reproductive Biology and Endocrinology, doi 10.1186/s12958-015-0032-1.

Alon, I., Guimon, J., \& Urbanos-Garrido, R. (2019). What to expect from assisted reproductive technologies? Experts' forecasts for the next two decades. Technological Forecasting and Social Change, Vol. 148, doi: org/10.1016/j.techfore.2019.119722.

Aslamkhan, M. (2015). Clinical Genetics and Genetic Counselling in Pakistan. Journal of Genes \& Cells, 1(2): p, 31-33, doi: 10.15562/gnc.17.

ASRM, P. C. (2015). Diagnostic evaluation of the infertile male: a committee opinion. Fertility and Sterility, 103(3), e18-25. doi: 10.1016/j.fertnstert.2014.12.103.

Babar, U. (2017). Monogenic Disorders: an Overview. International Journal of Advanced Research, 5(2) 1398-1424, doi: 10.21474/IJAR01/3294.

Bagheri, A., Moreno, J. D., \& Semplici, S. (2016). Global Bioethics: The Impact of the UNESCO International Bioethics Committee. Springer International Publishing, doi: 10.1007/978-3-31922650-7.

Batzer, F. R., \& Ravitsky, V. (2009). Preimplantation Genetic Diagnosis: Ethical Considerations. In V. Ravitsky, A. Fiester, \& A. L. Caplan, The Penn Center Guide to Bioethics. (pp. 339-354). New York: Springer .

Beck-Gernsheim, E. (2000). Health and Responosibility: From Social Change to Technological Change and Vice Versa. In B. Adam, U. Beck, \& J. Van Loon, The Risk Society and Beyond: Critical Issues for Social Theory (pp. 122-134). London: Sage.

Borup, M., Brown, N., Konrad, K., \& Van Lente, H. (2006). The Sociology of Expectations in Science and Technology. Technology Analysis \& Strategic Management, 18, 3/4, 285-298, doi: $10.1080=09537320600777002$.

Brown, N., \& Michael, M. (2003). A Sociology of Expectations: Retrospecting Prospects and Prospecting Retrospects. Technology Analysis \& Strategic Management, 15:1, 3-18, doi: 10.1080/0953732032000046024.

Buchanan, A., Brock, D. W., Daniels, N., \& Wikler, D. (2000). From Chance to Choice: Genetics and Justice. Cambridge University Press.

Cai, Q. F., Wan, F., Huang, R., \& Zhang, H. W. (2011). Factors Predicting the Cumulative Outcome of IVF/ICSI Treatment: a Multivariable Analysis of 2450 Patients. Human Reproduction, 9, 2532-40. doi: $10.1093 /$ humrep/der228. 
Casper, R., Haas, J., Hsieh, T., Bassil, R., \& Mehta, C. (2017). Recent Advances in in Vitro Fertilization. F1000Research, 6, 1616, doi: 10.12688/f1000research.11701.1.

CDC. (2018). Assisted Reproductive Technology - National Summary Report 2016. Atlanta, US,: Centers for Disease Control and Prevention, American Society for Reproductive Medicine, https://www.cdc.gov/art/pdf/2016-report/ART-2016-National-Summary-Report.pdf.

Dagan, E., Birenbaum-Carmeli, D., Friedman, E., \& Feldman, B. (2017). Performing and Declining PGD: Accounts of Jewish Israeli Women Who Carry a BRCA1/2 Mutation or Partners of Male Mutation Carriers. Journal of Genetic Counseling, 26, 5, doi: 10.1007/s10897-017-0087-6.

Dayé, C. (2018). How to Train Your Oracle: The Delphi Method and its Turbulent Youth in Operations Research and the Policy Sciences. Social Studies of Science, 48(6) 846-868, doi: $10.1177 / 0306312718798497$.

Di Stefano, G., Gambardella, A., \& Veronab, G. (2012). Technology Push and Demand Pull Perspectives in Innovation Studies: Current Findings and Future Research Directions. Research Policy, 41(8), 1283-1295, doi: 10.1016/j.respol.2012.03.021.

ESHRE. (2018). Assisted Reproductive Technology in Europe, 2014: Results Generated from European Registers by ESHRE. Human Reproduction, 33(9):1586-1601. doi: org/10.1093/humrep/dey242.

ESHRE Capri Workshop Group. (2014). Birth Defects and Congenital Health Risks in Children Conceived Through Assisted Reproduction Technology (ART): a Meeting Report. Journal of Assisted Reproduction and Genetics, 8, 947-58, doi: 10.1007/s10815-014-0255-7.

Evitt, N. H., Mascharak, S., \& Altman, R. B. (2015). Human Germline CRISPR-Cas Modification: Toward a Regulatory Framework. The American Journal of Bioethics, 15(12): 25-29, doi: 10.1080/15265161.2015.1104160.

Fauser, B. C., P., D., Diedrich, K., B. B., Bonduelle, M., Delemarre-van de Waal, H. A., . . Wells, D. (2014). Health Outcomes of Children Born After IVF/ICSI: a Review of Current Expert Opinion and Literature. Reproductive BioMedicine Online, 2, 162-82, doi: 10.1016/j.rbmo.2013.10.013.

Fauser, B., Boivin, J., Barri PN, T. B., \& Levy-Toledano, R. (2019). Beliefs, Attitudes and Funding of Assisted Reproductive Technology: Public Perception of Over 6,000 Respondents from 6 European Countries. PLOS ONE, 14(1), e0211150, doi: org/10.1371/journal.pone.0211150.

Fukuyama, F. (2002). Our Posthuman Future, Consequences of the Biotechnology Revolution. New York: Farrar, Straus and Giroux.

García, D., Vassena, R., Prat, A., \& Vernaeve, V. (2017). Poor Knowledge of Age-Related Fertility Decline and Assisted Reproduction Among Healthcare Professionals. Reproductive Biomedicine Online, 34, 1, 32-37, doi: org/10.1016/j.rbmo.2016.09.013. 
Garland-Thomson, R. (2015). Human Biodiversity Conservation: A Consensual Ethical Principle. The American Journal of Bioethics, 15(6):13-5. doi: 10.1080/15265161.2015.1028663.

Gomes de Oliveira, M. C., A. d., \& Nogueira-Martins, M. C. (2017). The Experience of the Bioethics Committee from a Public Hospital. Revista Bioetica, 25 (2): 338-47, doi: org/10.1590/198380422017252194.

Greely, H. T. (2016). The End of Sex and the Future of Human Reproduction. Cambridge: Harvard University Press.

Habermas, J. (2003). The Future of Human Nature. Cambridge: The translation polity press.

Hammarberg, K., Kirkman, M., \& de Lacey, S. (2016). Qualitative Research Methods:Wwhen to Use Them and How to Judge Them. Human Reproduction, 31, 3, 498-501, doi: 10.1093/humrep/dev334.

Harmon, S. H. (2016). Modernizing Biomedical Regulation: Foresight and Values in the Promotion of Responsible Research and Innovation. Journal of Law and the Biosciences, 680-686, doi: 10.1093/jlb/lsw053.

Harris, J. (2007). Enhancing Evolution, The Ethical Case of Making Better People. New Jersey: princeton University Presss.

Health Ministry of Israel. (2018). In Vitro Fertilization (IVF) Treatments 1990-2016. Israel: Facilities and Equipment Licensing Division, Health Information Division, https://www.health.gov.il/PublicationsFiles/IVF1990_2015.pdf.

Huxley, A. (1932). Brave New World. UK: Chatto \& Windus.

Inhorn, M. C., \& Patrizio, P. (2015). Infertility Around the Globe: New Thinking on Gender, Reproductive Technologies and Global Movements in the 21st Century. Human Reproduction Update, 21, 4, 411-426, doi: 10.1093/humupd/dmv016.

Jiahua, J., Xiangbin, Y., Yijun, L., \& Yumei, L. (2016). How users adopt healthcare information: An empirical study of an online Q\&A community. International Journal of Medical Informatics, 86, 91-103, doi: org/10.1016/j.ijmedinf.2015.11.002.

Jonas, H. (1984). The Imperative of Responsibility. University of Chicago.

Klitzman, R. (2008). Anticipating Issues Related to Increasing Preimplantation Genetic Diagnosis Use: a Research Agenda. Reproductive BioMedicine Online, 17, 1, 33-42, doi: 10.1016/S14726483(10)60188-5.

Knoepfler, P. (2016). GMO Sapiens, The Life-Changing Science of Designer Babies. Singapore: World Scientific Publishing.

Krimsky, S. (2019). Ten Ways in Which He Jiankui Violated Ethics. Nature Biotechnology, 37, 19, doi: org/10.1038/nbt.4337. 
La Marca, A., \& Sunkara, S. K. (2014). Individualization Of Controlled Ovarian Stimulation In IVF Using Ovarian Reserve Markers: From Theory To Practice. Human Reproduction Update, 20(1), 124-40, doi: 10.1093/humupd/dmt037.

Landeta, J., \& Barrutia, J. (2011). People Consultation to Construct the Future: A Delphi Application. International Journal of Forecasting, 27(1), 134-151, doi: .org/10.1016/j.ijforecast.2010.04.001.

Landeta, J., J., M., Ruíz, V., \& Galter, J. (2008). Results of a Delphi Survey in Drawing Up the Input-Output Tables for Catalonia. Technological Forecasting \& Social Change, 75(1), 32-56, doi: org/10.1016/j.techfore.2007.01.005.

Lewis, C. S. (1943). The Abolition of Man. New York: Oxford University Press.

Lippman, A. (1991). Prenatal Genetic Testing and Screening: Constructing Needs and Reinforcing Inequities. American Journal of Law and Medicine,, 17(1-2):15-50.

Lock, S. (1990). Towards a National Bioethics Committee. British Medical Journal, 300, 1149-1150, doil: 10.1136/bmj.300.6733.1149.

Lu, L., Lv, B., Huang, K., Xue, Z., Zhu, X., \& Fan, G. (2016). Recent Advances in Preimplantation Genetic Diagnosis and Screening. Journal of Assisted Reproduction and Genetics, 33, 9,1129-1134, doi: 10.1007/s10815-016-0750-0.

Martin, L. J. (2014). The World's Not Ready for This: Globalizing Selective Technologies. Science, Technology, \& Human Values, 39(3), 432-455, doi: 10.1177/0162243913516014.

Murphy, T. (2014). Im Defence of Prenatal Genetic Interventions. Bioethics, 28(7), 335-42, doi: 10.1111/j.1467-8519.2012.02009.x.

Nemet, G. F. (2009). Demand-Pull, Technology-Push, and Government-Led Incentives for NonIncremental Technical Change. Research Policy, 38(5) 700-709, doi: 10.1016/j.respol.2009.01.004.

Nuffield Council on Bioethics. (2018). Genome Editing and Human Reproduction: Social and Ethical Issues. London.

Orvieto, R., \& Gleicher, N. (2016). Should Preimplantation Genetic Screening (PGS) be Implemented to Routine IVF Practice? Journal of Assisted Reproduction and Genetics, 11, 1445-1448, doi: 10.1007/s10815-016-0801-6.

Paraskevopoulou, E. (2012). Non-Technological Regulatory Effects: Implications for Innovation and Innovation Policy. Research Policy, 41,6, 1058-1071, doi: org/10.1016/j.respol.2012.03.018.

Pavone, V. (2010). Genetic Testing, Geneticisation and Social Change: Insights from Genetic Experts in Spain. In W. Bernhard, \& W. Berger, Assessing Life: On the Organisation of Genetic Testing (pp. 101-132). Czech Republic: PBtisk s.r.o., P`ribram (CZ). 
Pavone, V., \& Arias, F. (2012). Beyond the Geneticization Thesis: The Political Economy of PGD/PGS in Spain. Science, Technology, \& Human Values, 37(3) 235-261, DOI: 10.1177/0162243911411195.

Ramsey, P. (1972). Shell we "Reproduce"? JAMA, 220 (10), 1346-1350; (11), 1481-1485.

Ravitsky, V. (2002). Genetics and Education: the Ethics of Shaping Human Identity. Mount Sinai Journal of Medicine, 69(5):312-6. .

Ribeiro, B. E., \& Quintanilla, M. A. (2015). Transitions in Biofuel Technologies: An Appraisal of the Social Impacts of Cellulosic Ethanol Using the Delphi Method. Technological Forecasting \& Social Change, 92, 53-68, do: 10.1016/j.techfore.2014.11.006.

Rogers, E. M. (1983). Diffusion of Innovation. New York: The Free Press.

Rommetveit, K. (2011). Genetic Enhancement, Futures Tense. Futures, 43, 76-85, doi: 10.1016/j.futures.2010.10.014.

Sandel, M. J. (2004). The Case Against Perfection. The Atlantic, 293, 3, 51-62.

Second International Summit on Human Genome Editing. (2018a). Genes, Genomes, and Genetic Variation. (pp. Day 1, Session 1). Hong Kong: The National Academies of Sciences Engineering Medicine, http://www.nationalacademies.org/gene-editing/2nd_summit/first_day/index.htm.

Second International Summit on Human Genome Editing. (2018b). Global Perspectives: Somatic and Germline Therapy, Prevention, and Enhancement Applications, Identifying Basic Principles for Moving Forward. (pp. Day 3, Session 1, and 2). Hong Kong: The National Academies of Sciences Engineering Medicine, http://www.nationalacademies.org/geneediting/2nd_summit/third_day/index.htm.

SEF. (2010). Registro de la Sociedad Española de Fertilidad: Técnicas de Reproducción Asistida (IA y FIV/ICSI). Año 2.010. https://www.registrosef.com/public/Docs/sef2010_IAFIV.pdf: Sociedad Espanola de Fertilidad.

SEF. (2016). Registro Nacional de Actividad - Técnicas de Reproducción Asistida , Informe estadístico de Técnicas de Reproducción Asistida 2016. Sociedad Espanola de Fertilidad.

Shalev, C., \& Hashiloni-Dolev, Y. (2011). Bioethics Governance in Israel: an Expert Regime. Indian Journal of Medical Ethics, 8, 3, https://ijme.in/articles/bioethics-governance-in-israel-an-expertregime/?galley=pdf.

Shulman, C., \& Bostrom, N. (2014). Embryo Selection for Cognitive Enhancement: Curiosity or Gamechanger? Global Policy, 5(1) 85-92, doi: 10.1111/1758-5899.12123 .

Silver, M. L. (1997). Remaking Eden: How Genetic Engineering and Cloning Will Transform the American Family. New York: Harper Perennial. 
Skirton et al. (2013). A Delphi Study to Determine the European Core Curriculum for Master Programmes in Genetic Counselling. European Journal of Human Genetics, 21, 1060-1066, doi: 10.1038/ejhg.2012.302.

Sobotka, T. (2016). Childlessness in Europe: Reconstructing Long-Term Trends Among Women Born in 1900-1972. In M. Kreyenfeld, \& D. Konietzka, Childlessness in Europe: Contexts, Causes, and Consequences (pp. 17-50). Berlin: Springer Open.

Swanson, A., Lau, E., \& Strawn, E. (2007). Preimplantation Genetic Diagnosis: Technology and Clinical Applications. Wisconsin Medical Journal, 106(3):145-51.

Tarkkala, H., Helén, I., \& Snell, K. (2018). From Health to Wealth: The Future of Personalized Medicine in the Making. Futures, doi: org/10.1016/j.futures.2018.06.004.

ten Have, H. A. (2001). Genetics and Culture: The Geneticization Thesis. Medicine, Health Care and Philosophy, 4: 295-304.

van Dijke, I., Bosch, L., Bredenoord, A., Cornel, M., Repping, S., \& Hendriks, S. (2018). The ethics of clinical applications of germline genome modification: a systematic review of reasons. Human Reproduction, Vol.33, No.9 pp. 1777-1796, doi:10.1093/humrep/dey257.

Von der Gracht, H. A. (2012). Consensus Measurement in Delphi Studies Review and Implications for Future Quality Assurance. Technological Forecasting \& Social Change, 79(8), 1525-1536, doi: 10.1016/j.techfore.2012.04.013.

Wang, Z. (2014). Automatic Zona Pellucida Dissection Position Selection for Embryo Biopsy in Preimplantation Genetic Diagnosis. International Conference on Robotics and Biomimetics (pp. 475480). Bali: IEEE.

Zuckerman, S., Zeevi, D. A., Gooldin, S., \& Altarescu, G. (2017). Acceptable Applications of Preimplantation Genetic Diagnosis (PGD) among Israeli PGD Users. European Journal of Human Genetics, 25, 1113-1117, Doi: 10.1038/ejhg.2017.113. 